\title{
Infrared continuum sizes of Be star disks
}

\author{
Douglas R. Gies ${ }^{1}$, Yamina N. Touhami ${ }^{1}$ and Gail H. Schaefer ${ }^{2}$ \\ ${ }^{1}$ Center for High Angular Resolution Astronomy and Department of Physics and Astronomy, \\ Georgia State University, P. O. Box 4106, Atlanta, GA 30302-4106, U.S.A. \\ email: gies@chara.gsu.edu, yamina@chara.gsu.edu \\ ${ }^{2}$ Georgia State University, CHARA Array, P. O. Box 48, Mount Wilson, CA 91023, U.S.A. \\ email: schaefer@chara-array.org
}

\begin{abstract}
Long baseline interferometry now offers us the opportunity to measure the dimensions of Be star circumstellar disks across the spectrum. This includes the near-infrared continuum where the emission is dominated by bound-free and free-free emission from the ionized disk gas. Here we present the results of calculations of the disk sizes and continuum flux excesses for a simple version of the viscous decretion model of the disk. We compare these results to recent $18 \mu \mathrm{m}$ flux measurements from the AKARI infrared satellite all-sky survey.
\end{abstract}

Keywords. techniques: interferometric, circumstellar matter, stars: emission-line, Be

Be stars are bright in the infrared due to emission from the ionized gas in their circumstellar disks (Waters et al. 1987; Dougherty et al. 1994; Zhang et al. 2005). It is now possible to angularly resolve the disks of relatively nearby Be stars with long baseline interferometers like the CHARA Array and VLTI (Gies et al. 2007; Meilland et al. 2007). Since the IR flux excess depends on both the source function (dependent on temperature) and projected disk size, a combined interferometric and flux excess analysis can yield valuable information on the disk gas temperature. Gies et al. (2007) presented a realization of the viscous decretion model for isothermal disks of Be stars that they used to create model images, interferometric visibilities, and IR fluxes. The model is based upon four main parameters: the gas base density $\rho_{0}$, the radial density exponent $n$, the disk inclination $i$, and the disk-to-star temperature ratio $T_{d} / T_{\text {eff }}$. Here we use the model to calculate the disk IR fluxes from 1.7 to $18 \mu \mathrm{m}$ in wavelength.

We begin with a default model with a central star with $T_{\text {eff }}=30 \mathrm{kK}, R / R_{\odot}=10$, and $M / M_{\odot}=15.5$. The adopted disk parameters are $n=3, i=45^{\circ}, T_{d} / T_{\text {eff }}=2 / 3$, and an outer boundary disk radius $R_{\text {out }} / R_{\star}=21.4$. We show in Figure 1 the predicted $18 \mu \mathrm{m}$ flux excess $E^{\star}\left(V^{\star}-m_{\lambda}\right)=2.5 \log \left(1+F_{d} / F_{\star}\right)$ and ratio of the disk radius (HWHM) to stellar radius along the projected major axis of the disk. These are plotted for a range in base density of $\log \rho_{0}=-12.0$ to -9.7 (units of $\mathrm{g} \mathrm{cm}^{-3}$ ). Also shown are several other model sequences made by varying one of the disk parameters. The position in the diagram is most sensitive to disk density and inclination, but there is a systematic displacement between models with different assumed $T_{d} / T_{\text {eff }}$, which indicates that the near-IR fluxes and interferometric diameters can be used to estimate disk temperature (especially for dense, large disks).

We can test the model predictions through a comparison of the the 2MASS and AKARI fluxes (Ita et al. 2010) for a sample of Be stars from Dougherty et al. (1994). We determined for each star an observational excess pegged to the $V$-band flux

$$
E^{\star}\left(V^{\star}-m_{\lambda}\right)=V-m_{\lambda}-E(B-V) \times\left(3.10-R_{\lambda}\right)-\left(V-m_{\lambda}\right)(\text { Kurucz })
$$

where the ratio of interstellar extinction to reddening is $R_{\lambda}=A_{\lambda} / E(B-V)$ and $(V-$ $\left.m_{\lambda}\right)$ (Kurucz) is the intrinsic stellar colour derived from monochromatic sampling of flux 


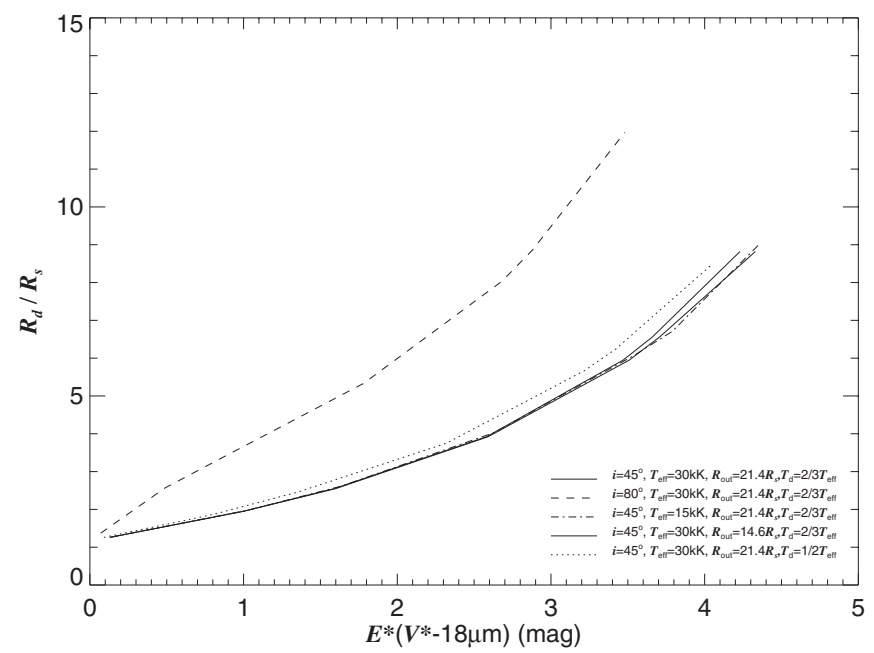

Figure 1. A plot of the disk HWHM versus the $18 \mu \mathrm{m}$ flux excess for several models.

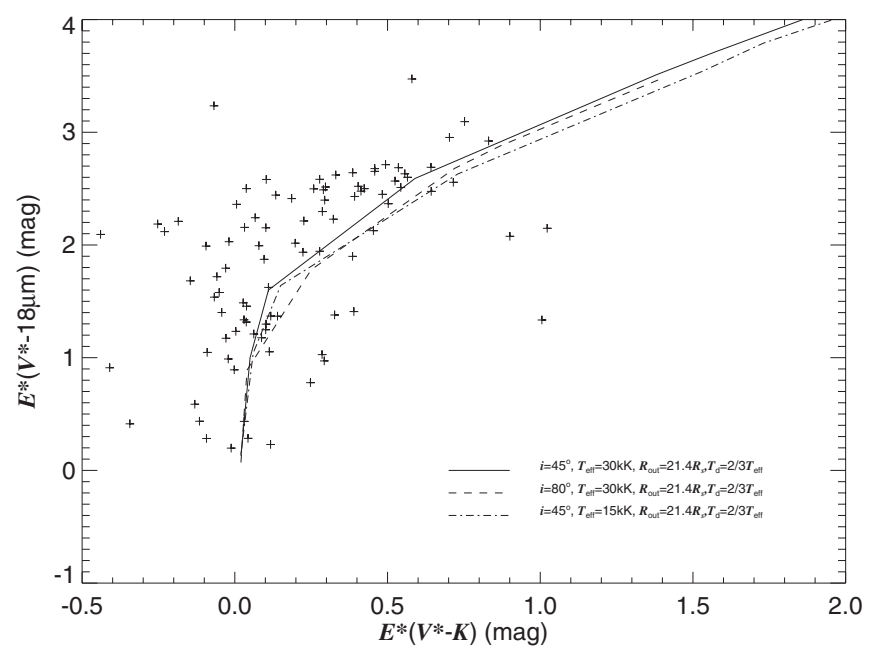

Figure 2. A comparison of 2MASS $K_{s}$-band and AKARI $18 \mu \mathrm{m}$ flux excesses with models.

ratios of model spectra with a Vega spectrum from R. L. Kurucz. The equivalence of the model and observed colour excesses is based upon the assumption that the disk contributes no flux in the $V$-band, and this neglect may lead to somewhat negative colour excesses for the dense disk cases. A comparison of the $K_{s}$ and $18 \mu \mathrm{m}$ flux excesses is shown in Figure 2. The overall agreement suggests that the viscous decretion disk model provides a satisfactory description of the near-IR flux excesses.

\section{References}

Dougherty, S. M., Waters, L. B. F. M., Burki, G., Cote, J. et al. 1994, A\& $A, 290,609$

Gies, D. R., Bagnuolo, Jr., W. G., Baines, E. K., ten Brummelaar, T. A. et al. 2007, ApJ, 654, 527

Ita, Y., Matsuura, M., Ishihara, D., Oyabu, S. et al. 2010, $A \mathscr{E} A$, 514, A2

Meilland, A., Stee, P., Vannier, M., Millour, F. et al. 2007, A\&A, 464, 59

Waters, L. B. F. M., Cote, J. \& Lamers, H. J. G. L. M. 1987, A\& A, 185, 206

Zhang, P., Chen, P. S. \& Yang, H. T. 2005, New Astron., 10, 325 